ORIGINAL ARTICLE

\title{
Multi Drug Resistance (MDR) Urinary Tract Infection: An Evidence Based Study
}

\author{
NASIR ORAKZAI ${ }^{1}$, LIAQAT ALI ${ }^{2}$, MAJID KHAN KAKAKHEL ${ }^{3}$, ARSHAD $^{4}$, FAIZA HAYAT ${ }^{5}$, IHSANULLAH KHAN ${ }^{6}$ \\ ${ }^{1}$ Professor of Urology Rehman Medical Institute former Director Institute of Kidney Diseases HMC Peshawar \\ ${ }^{2}$ Professor of Urology Chairman Department of Urology and Transplant Institute of Kidney Diseases HMC Peshawar \\ ${ }^{3}$ Medical officer Institute of Kidney Diseases HMC Peshawar \\ ${ }^{4}$ Medical officer Institute of Kidney Diseases HMC Peshawar \\ ${ }^{5}$ Specialist Registrar Urology Institute of Kidney Diseases Hayatabad Medical Complex Peshawar \\ ${ }^{6}$ Specialist Registrar Urology Institute of Kidney Diseases Hayatabad Medical Complex Peshawar \\ Correspondence to: Dr Liaqat Ali, Email: liaqat_99@yahoo.com cell no: 00340966665
}

\begin{abstract}
Background: Urinary tract infections are the most frequently reported infections that drive the use of antibiotics around the world. UTI is the 4th most common healthcare-associated infection. Multidrug-resistant (MDR) organisms are predominantly bacteria that are resistant to one or more classes of antimicrobials. The increasing rise in the incidence of MDR-UTI has resulted in increased morbidity, mortality, and treatment cost of the patients. Thus, it is important to highlight the magnitude of the problem, identify the risk factors that result in MDR-UTI, and to take appropriate measures to control its occurrence.

Objective: To determine the magnitude of the multidrug-resistant bacteria, their antibiotic-resistant profile, andtheir effect on the treatment cost of the patients

Methods: It is a descriptive study conducted in the Department of Urology at the Institute of Kidney Diseases (IKD) from Jan 2019 till 30th March 2020. A total of 54 patients with multi-drug resistant UTI were included in the study irrespective of age and gender. All the data was recorded on a structured pro-forma and was analyzed on SPSS.

Results: A total of 3190 patients were operated on from Jan 2019 till 30th March 2020. Out of which 54 patients $(1.6 \%)$ developed MDR-UTI. Among them,38 were male and 16 females. The mean age of the patients was $41 \pm$ 18.4. Urolithiasis with infections was found most frequent, in $32(59.3 \%)$ patients. All patients were on broadspectrum oral antibiotics and had a history of urethral catheterization before the development of MDR-UTI. The most common procedure was Emergency cystoscopy and DJ stent 15 (27.8\%). Followed by Percutaneous nephrostomy in $8(14.8 \%)$. Regarding co-morbidities, 38(68.5\%) patients had none, 3 patients had diabetes and 6 patients were having Diabetes and Hypertension. Pseudomonas aeruginosa was found most frequent microorganisms in $34(63 \%)$ patients while E.coli in $10(18.5 \%)$ and Klebsiella in $5(9.3 \%)$ patients. Colistin was found sensitive in 36 patients $(66.7 \%)$. The mean hospital stay in MDR-UTI is $9.28 \pm 5.17$ days as compared to 2.1 days in routine cases. Approximately a 4 -fold increase was observed in medicines alone in the management of MDR UTI. We recorded 1 mortality (1.9\%), case of MDR urosepsis. Linear regression revealed previous use of antibiotics; catheterization, old age, and endo-urological procedures in an emergency as independent risk factors for MDR-UTI.

Conclusion: MDR-uti is an emerging local problem. pseudomonas aeruginosa is the most frequently found microorganism in the present setup. it is associated with significant morbidity and very high treatment cost.

Keywords: Urinary Tract Infection, Multidrug Resistance, Micro-Organism, Urology, Antimicrobials
\end{abstract}

\section{INTRODUCTION}

Urinary tract Infections (UTI) are the most frequently reported infections that drive the use of antibiotics around the world [1]. UTI is the 4th most common type of healthcare-associated infection [2]. It accounts for all most 8.3 million physician visits, 1million emergency department visits, and 100,000 hospitalizations per annum, with an estimated cost of $\$ 1$ billion per annum in the United States [3]. The vast majority of UTIs arise in female outpatients, many of whom are treated empirically by their primary physicians $[3,4,5]$.

Antimicrobial resistance patterns are continually evolving. The prevalence of multidrug resistance among isolates of E. coli in the United States (7.1\%) suggests that these phenotypes be monitored and empirical treatment of UTI is updated. Fluoroquinolones use and urinary tract abnormalities are associated with resistant UTIs in urology [6].Globally more than 100,000 deaths each year are attributable to drug-resistant infections [7], a number that is expected to increase due to the dearth of new antibiotics in development. While urologists confidently prescribe the choice of treatment without having accurate knowledge related to local antibiotic resistance, often choosing antibiotics that are not consistent with published guidelines [8].

It is, therefore, necessary to conduct properly designed regional surveillance studies, which would help to identify factors that place patients at increased risk for a MDR infection. This will ultimately lead to controlling the further spread of such infections, lessening the morbidity and mortality because of these infections The purpose of this study is to highlight the magnitude of the MDR UTIs in our practice as this issue has not been addressed yet, which leaves our urologist naïve and unprepared for a very serious looming problem.

\section{MATERIALS AND METHODS}

Following the institute of kidney diseases and renal transplant research ethics board approval this 
observational study identified all patients with multi-drug resistant UTI irrespective of age and gender. The patients data was accessed retrospectively from hospital records and collected on structured Pro-forma from Jan 2019 to March 2020. A total of 54 patients of MDR-UTI were identified. The data collected included Organism cultured, Antimicrobial Sensitivity, Resistance, Diagnosis, and the type of procedure done. Statistical analyses were performed with IBM $®$ SPSS $\AA$, version 20.0.

\section{RESULTS}

Total number of 3190 patients were operated from Jan, 2019 till 30th March, 2020. Out of which 54 patient (1.6\%) developed MDR-UTI with mean age of $41 \pm 18.4$ years. 38 were male and 16 female. The Organisms Cultured were Pseudomonas aeruginosa $(n=34,63 \%)$, E. Coli $(n=10$, $18.5 \%)$, Klebsiella $(n=5,9.3 \%)$, Proidencia $(n=1,1.9 \%)$, Enterobacter $(n=1,1.9 \%), \quad$ Enterococcus $(n=1,1.9 \%)$, Serratia Marcescens $(n=1,1.9 \%)$, Staphylococcus Aureus $(n=1,1.9 \%)$. Antimicrobial Sensitivity to colistin $(n=36$, $66 \%)$, Carbapenem $(n=7,13 \%)$, Aminoglycoside $(n=4$, $7.4 \%)$, fosfomycin $(n=2,3.7 \%)$, Vancomycin $(n=1,1.9 \%)$, Tigecycline $(n=1,1.9 \%)$ was found while antimicrobial Sensitivity to none was $(n=3,5.6 \%)$. Antimicrobial Resistance to Fluoroquinolones and Trimethoprim + Sulfamethoxazol was $100 \%$. The underlying pathology included urolithiasis $(\mathrm{n}=32,59.3 \%)$, isolated UTI without definite pathology $(n=15,27.81 \%)$, NGB $(n=3,5.6 \%)$, TCC Bladder in $2(n=2,3.7 \%)$, Infection with Enlarged Prostate and obstructive uropathy secondary to RPF $(n=1,1.9 \%)$ each. The Procedures done in these patients were found Emergency Cystoscopy plus DJS ( $n=15,27.8 \%), P C N$ $(n=8,14.8 \%)$, URS plus ICL and DJS $(n=6,11.1), P C N L$ $(n=6,11.1 \%)$, TURP $(n=4,7.4 \%)$, open Surgery $(n=4$, $7.4 \%)$, RIRS $(n=3,5.6 \%)$, ESWL $(n=3,5.6 \%)$, CIC $(n=2$, $3.7 \%)$, TURBT $(n=1,1.9 \%)$, Cystoscopy plus Urethral Dilatation ( $n=1,1.9 \%)$ and Cystoscopy Followed by Ureterocoele incision $(n=1,1.9 \%)$. The mean hospital stay in MDR-UTI was 9.28 5.17 days as compared to 2.1 days in routine cases. We recorded 1 mortality $(1.9 \%)$ in patient with MDR Urosepsis. No Co-Morbidity was recorded in $38(68.5 \%)$, while Diabetes Mellitus and Hypertension in 6 patients $(11.1 \%)$, Hypertension in $6(11.1 \%)$, Diabetes in 3 (5.6\%), IHD in 1 (1.\%) and Hepatitis B Virus in $1(1.9 \%)$ was recorded.

Figure No 1: Frequency of organisms

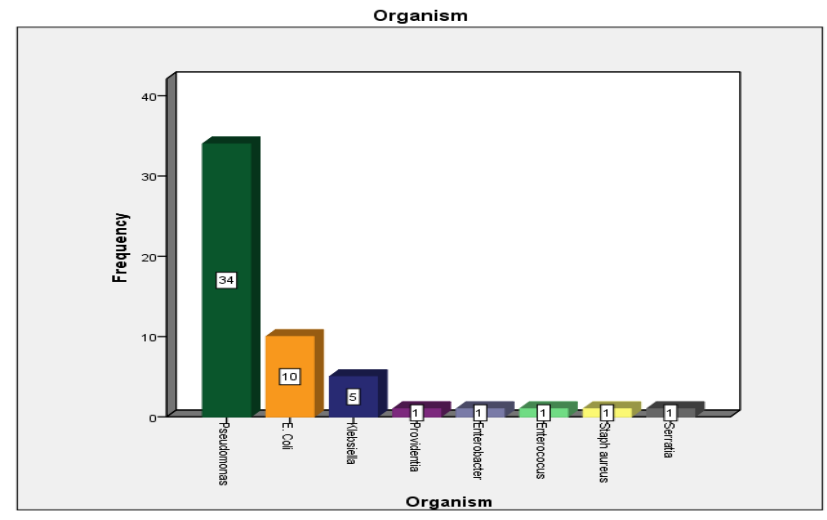

Figure No 2: Different diagnosis among all the included patients

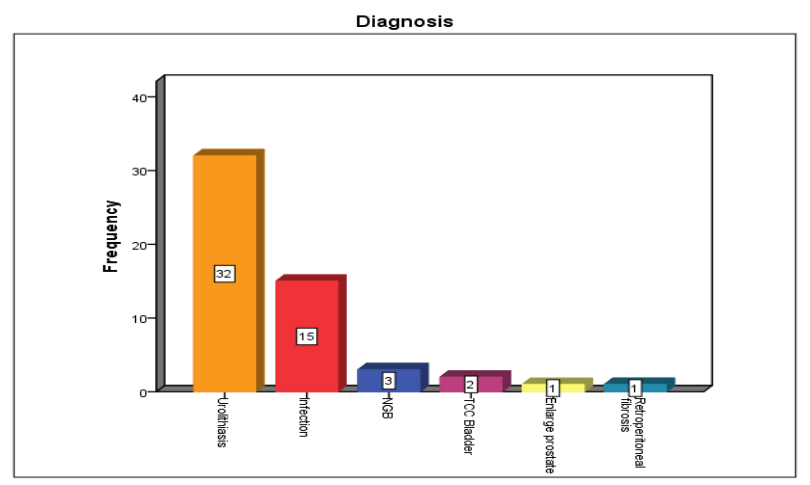

Table No 1: Antimicrobial sensitivity

\begin{tabular}{|l|l|l|}
\hline Sensitive to & Frequency & Percent \\
\hline Formula & 36 & 66.7 \\
\hline Colistin & 1 & 1.9 \\
Tigecycline & 7 & 13.0 \\
Carbapenem & 4 & 7.4 \\
Aminoglycoside & 3 & 5.6 \\
None & 2 & 3.7 \\
Fosfomycin & 1 & 1.9 \\
Vancomycin & 54 & 100.0 \\
\hline Total & & \\
\hline
\end{tabular}

Figure No 3: Frequency of Procedures

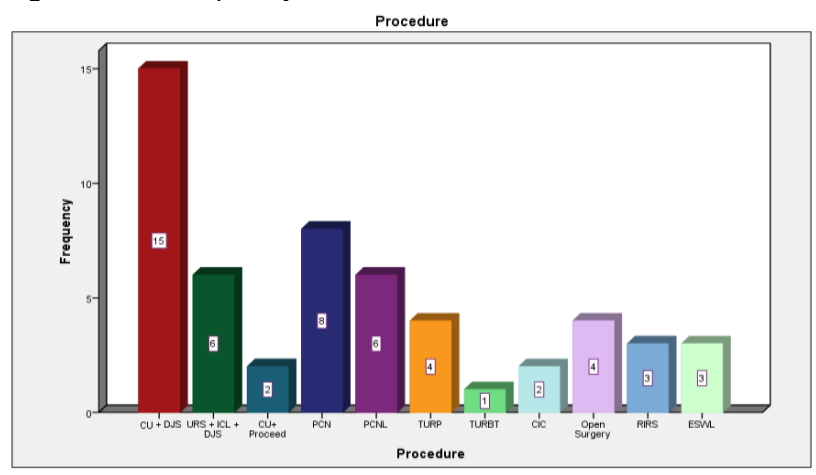

\section{DISCUSSION}

The emergence of high rates of antibiotic-resistant organisms and MDR-phenotypes causing urinary tract infections has become a public health concern worldwide. In our study, the most frequently cultured organism in MDR-UTI isPseudomonas aeruginosa ( $P$. aeruginosa.) with an incidence of $63 \%$ followed by $E$-coli noted to be $18.5 \%$. Our statistics in terms of the predominant organism and its incidence rate appears to differ from international studies. In the study by Durgesh D. Wasnik et al. [9], the incidence ofP. aeruginosa was $15 \%$ and that of E.Coli $31 \%$, while the prevalence of $\mathrm{P}$. aeruginosa. in another European study was $6.9 \%$. (11). In Kashmir, P.aeruginosa. infection accounted for $7.6 \%$ of the urine samples (12) while in Nepal, P. Aeruginosa accounted for $1.20 \%$ of the total cultures (13).A study from India showed a prevalence of $P$. AERUGINOSA to be $9.3 \%$ (14) and in a Palestinian study(15) $9 \%$ of the P.aeruginosagrowth was noted in the cultures.

One study in Pakistan showed E. Coli the most predominant organism (42.9\%) while P. aeruginosa in only 
5.4\% [10]. In another Pakistani study, the prevalence of the P.aeruginosa was noted to be $9.2 \%$ and E-Coli $3.27 \%(16,17)$. It shows the $P$. aeruginosa Is the commonest MDR organism in our practice, contrary to the findings of other studies, in which E-coli appears as the leading cause of MDRUTI. Knowing that all of our patients had urethral procedures performed in hospitals, we can infer that the infection in our studied patients had hospitalacquired organisms.

In contrast with global trends, our results reveal most of our patientshaving multidrug-resistant urinary tract infections being male rather than females [18-21].This may be due to the lower number of female patients presenting to our urology set up.In our overall cultured organisms, $66 \%$ were sensitiveto colistinand13\% to Carbapenem. P.aeruginosa was mostly sensitive to colistin(79.4\%)and Ecoli to Carbapenems (50\%). Studies in India showed P. aeruginosa species were $65 \%$ susceptible to colistin. [22]Mahfoud $M$ et al noticed $89.1 \%$ response of $P$. aeruginosa to colistin [23]. Thestudy by Toniolo A. et al says Highly aggressive multi-drug-resistant (MDR) $P$. aeruginosa strains were widespread, although colistin remained effective in $100 \%$ of cases. [24].The relatively higher Colistin resistance noted in Pakistan and India could reflect the indiscreet use of the antibiotics, even that of the last line drugs among them. The most common misuse of such medications occurs when infections are treated empirically, in improper dosage, and with no clear indications e.g. in patients with long-term symptomatic catheterized patients, etc.In another study by Falagas ME et al, they mentioned that in Recent studies of patients who received intravenous colistin for the treatment of serious $P$. aeruginosa infections of various types, including urinary tract infections, colistin is both effective and has less toxic in contrast to previous studies [27]. In a study in India, they found $98.9 \%$ of E. Coli Strains Sensitive to Carbapenems. [25]. Zhang, $H$. et al in 2014 and 2015 identified Susceptibilities of Escherichia coli in UTI strains to carbapenems of $>90 \%$ [26]. Additionally, ourstudy also showed $100 \%$ isolates resistance to Fluoroquinolones and trimethoprim-sulfamethoxazole (TMPSXT), which is recommended as the first choice for UTI treatment [21]. Previous studies reported similar results [21, 28]Three organisms identified wereresistant to all available antimicrobials, out of the one case is E. coli (ESBL) and two cases of pseudomonas aeruginosa. The findings of our study when compared to the quoted regional studies, points out that during the last 10 years, there has been an increased bacterial resistance to fluoroquinolones

In a study by Mahfoud $M$ et al resistance of Pseudomonas aeruginosa to TMP + SMX was $88.3 \%$ [23].Aypak $C$ et al noticed Resistance to TMP-SMX(41.1\%) and to Fluoroquinolones it was (25.2\%)[29]. Mandal, J et al identified Seventy-three percent of all E.coli isolates were found to be resistant to ciprofloxacin. Ciprofloxacin resistance was comparatively less in Pseudomonas aeruginosa.[30]. These rates of resistance vary, depending upon whether the study sample consists of primarily simple UTI or complicated UTI, hospital or community-acquired.

Presently, recommended antibiotics against Enterobacteriaceae are fosfomycin, carbapenems, and aminoglycosides. Quinolones, trimethoprim, and sulfamethoxazole are inappropriate for empirical therapy due to the high incidence of resistance. Among aminoglycosides, Amikacin is more effective.It may very effective, in complicated cases when usingit in combination with fosfomycin and Carbapenems [31]

It has been observed that our urologists tend to treat suspected UTIs empirically. The reasons cited by them for this practice is, poor patient compliance to do cultures for monetary and logistic reasons.

Such practice of the clinicians, adds to other factors outlined above, in predisposing to the emergence of resistant bacterial strains.

Our in-patient hospital stay for UTI patients is more than 3 times as compared to other admitted cases. All these patients had failed to respond to previously administered antibiotics and thus needed intravenous lastline antibiotics. Urine culture and sensitivity were performed when they were not on antibiotics. The increase in the duration of hospital stay strained our already overstretched resources and understandably seriously affected patients financially and psychologically.

\section{CONCLUSION}

Multi resistant pseudomonas aeruginosa is the most frequently found microorganism in our present setup. This organism is sensitive to limited antibiotics, mostly to colistin as is the case in neighboring india, which has somewhat similar standards of medical practices. The high incidence of the reported bacteriaresulted in a longer duration of treatment, prolonged hospital stay clearly suggests overstretching of our scarce public-sector hospital resources, which is of utmost importance to bear in mind, especially with the ever-increasing patient workload. to make certain the causes for reported MDR pseudomonas aeruginosa infections in our study, its observed predominance in our males patients, the possible psychological impact on the affected patients by it and also to compare our findings with that of the similar regional hospitals, more in-depth and broad-based study is required.

\section{REFERENCES}

1. Allen U.D., MacDonald N., Fuite L., Chan F., Stephens D. Risk factors for resistance to first-line antimicrobials among urinary tract isolates of Escherichia coli in children. CMAJ. 1999;160(10):14361440. [PMC free article] [PubMed] [Google Scholar] [Ref list]

2. Arslan H., Azap O.K., Ergonul O., Timurkaynak F. Risk factors for ciprofloxacin resistance among Escherichia coli strains isolated from community-acquired urinary tract infections in Turkey. J. Antimicrob. Chemother. 2005;56:914918. [PubMed] [Google Scholar] [Ref list]

3. Foxman B. Epidemiology of urinary tract infections: Incidence, morbidity, and economic costs. Am J Med. 2002;113(suppl 1A):5S-13S

4. Gupta, K. A., D. Scholes, and W. E. Stamm. 1999. Increasing prevalence of antimicrobial resistance among uropathogens causing acute uncomplicated cystitis in women. JAMA 281:736738.

5. Hooton, T. M., and W. E. Stamm. 1997. Diagnosis and treatment of uncomplicated urinary tract infection. Infect. Dis. Clin. N. Am. 11:551581. 
6. Ena j, Amador C, Martinez $\mathrm{C}$ et al: Risk factors for acquisition of urinary tract infections caused by ciprofloxacin resistant Escherichia coli. J Uro 1995; 153: 117.

7. O'Neill J, Adams E, Arinaminpathy $\mathrm{N}$ et al: Tackling DrugResistant Infections globally: Final Report and Recommendations. The review on antimicrobial resistance, May 2016.

8. Lebentrau S, Gilfrich C, Vetterlein MW, Schumacher $\mathrm{H}$, Spachmann PJ, Brookman-May SD, et al. Impact of the medical specialty on knowledge regarding multidrugresistant organisms and strategies toward antimicrobial stewardship. Int Urol Nephrol. 2017;49(8):1311-8.

9. Wasnik DD. Prevalence and antibacterial susceptibility pattern of urinary tract infection Causing Human Pathogenic Bacteria. Asian J Biomed Pharm Sci. 2012;2(15):13. doi:10.15272/ajbps.v2i15.130.

10. Shah DA, Wasim S, Abdullah FE. Antibiotic resistance pattern of Pseudomonas aeruginosa aeruginosa isolated from urine samples of Urinary Tract Infections patients in Karachi, Pakistan. Pak J Med Sci 2015;31(2):341-345.

11. Bouza E, San Juan R, Muñoz P, Voss A, Kluytmans J. Cooperative Group of the European Study Group on Nosocomial Infections: A European perspective on nosocomial urinary tract infections $I$. Report on the microbiology workload, etiology and antimicrobial susceptibility (ESGNI-003 study). European Study Group on Nosocomial Infections. Clin Microbiol Infect. 2001;7(10):523531.

12. Ahmad S. Pattern of urinary tract infection in Kashmir and antimicrobial susceptibility. Bangladesh Med Res Counc Bull. 2012;38(3):79-83.

13. Khatri B, Basnyat S, Karki A, Poudel A, Shrestha B. Etiology and antimicrobial susceptibility pattern of bacterial pathogens from urinary tract infection. Nepal Med Coll J. 2012;14(2):129-132

14. Manjunath GN, Prakash R, Annam V, Shetty K. Changing trends in the spectrum of antimicrobial drug resistance pattern of uropathogens isolated from hospitals and community patients with urinary tract infections in Tumkur and Bangalore. Int J Biol Med Res. 2011;2(2):504-507.

15. Zakaria EA. Increasing Ciprofloxacin resistance among prevalent urinary tract bacterial isolates in Gaza Strip, Palestine. J Biomed Biotechnol. 2005;(3):238-241. doi:10.1155/JBB.2005.238

16. Gul N, Mujahid TY, Ahmad S. Isolation, Identification and Antibiotic Resistance Profile of Indigenous Bacterial Isolates from Urinary Tract Infection Patients. Pak J Biol Sci. 2004;7(12):2051-2054.

17. Paryani JP, Memon SR, Rajpar ZH, Shah SA. Pattern and Sensitivity of Microorganisms Causing Urinary Tract Infection at Teaching Hospital. JLUMHS. 2012;11(2):97-100.

18. Dehbanipour R, Rastaghi S, Sedighi M, Maleki N, Faghri J. High prevalence of multidrug-resistance uropathogenic Escherichia coli strains, Isfahan, Iran. J Nat Sci Biol Med. 2016;7(1):226

19. Bouchillon $\mathrm{S}$, Hoban DJ, Badal R, Hawser S. Fluoroquinolone resistance among gram-negative urinary tract pathogens: global smart program results, 20092010. Open Microbiol J. 2012;6:748.

20. Tabasi M, Asadi Karam MR, Habibi M, Yekaninejad MS, Bouzari S. Pheno typic assays to determine virulence factors of uropathogenic Escherichia coli (UPEC) isolates and their correlation with antibiotic resistance rattern. Osong Public Health Res Perspect. 2015;6(4):2618.

21. Ali I, Rafaque S, Ahmed S, Malik JI, Dasti P. Prevalence of multi-drug resist ant uropathogenic Escherichia coli in Potohar region of Pakistan. Asian Pac J Trop Biomed. 2016;6(1):606.

22. Sultan A, Rizvi M, Khan F, Sami H, Shukla I, Khan HM. Increasing antimicrobial resistance among uropathogens: Is fosfomycin the answer? Urol Ann. 2015;7(1):26-30.

23. Mahfoud M, Al Najjar M, Hamzeh AR. Multidrug resistance in Pseudomonas aeruginosa aeruginosa isolated from nosocomial respiratory and urinary infections in Aleppo, Syria. The Journal of Infection in Developing Countries. 2015 Feb 19;9(02):210-3.

24. Toniolo A, Endimiani A, Luzzaro F. Microbiology of postoperative infections. Surg Infect (Larchmt). 2006;7 Suppl 2:S13-6.

25. Niranjan V, Malini A. Antimicrobial resistance pattern in Escherichia coli causing urinary tract infection among inpatients. The Indian journal of medical research. 2014 Jun;139(6):945

26. Zhang, H., Kong, H., Yu, Y., Wu, A., Duan, Q., Jiang, X., Zhang, S., Sun, Z., Ni, Y., Wang, W., Wang, Y., Liao, K., Li, H., Yang, C., Huang, W., Gui, B., Shan, B., Badal, R., Yang, Q., \& Xu, Y. (2018). Carbapenem susceptibilities of Gramnegative pathogens in intra-abdominal and urinary tract infections: updated report of SMART 2015 in China. BMC infectious diseases, 18(1), https://doi.org/10.1186/s12879-018-3405-1

27. Falagas ME, Kasiakou SK. Colistin: the revival of polymyxins for the management of multidrug-resistant gram-negative bacterial infections [published correction appears in Clin Infect Dis. 2006 Jun 15;42(12):1819. Dosage error in article text]. Clin Infect Dis. 2005;40(9):1333-1341. doi:10.1086/429323

28. Paniagua-Contreras GL, Monroy-Perez E, RodriguezMoctezuma JR, Dominguez-Trejo P, Vaca-Paniagua F, Vaca $S$. Virulence factors, antibiotic resistance phenotypes and Oserogroups of Escherichia coli strains isolated from community-acquired urinary tract infection patients in Mexico. J Microbiol Immunol Infect. 2017;50(4):47885.

29. Aypak C, Altunsoy A, Düzgün N. Empiric antibiotic therapy in acute uncomplicated urinary tract infections and fluoroquinolone resistance: a prospective observational study. Annals of Clinical Microbiology and Antimicrobials. 2009;8(1):27.

30. Mandal, J., Acharya, N. S., Buddhapriya, D., \& Parija, S. C. (2012). Antibiotic resistance pattern among common bacterial uropathogens with a special reference to ciprofloxacin resistant Escherichia coli. The Indian journal of medical research, 136(5), 842849.

31. Yekani, M., Baghi, H. B., Sefidan, F. Y., Azargun, R., Memar, M. Y., \& Ghotaslou, R. (2018). The rates of quinolone, trimethoprim/sulfamethoxazole and aminoglycoside resistance among Enterobacteriaceae isolated from urinary tract infections in Azerbaijan, Iran. GMS hygiene and infection control, 13, Doc07. https://doi.org/10.3205/dgkh000313 